\title{
LXXIV. On the Existence of absolute criteria for determining the roots of numerical equations
}

\section{J.J. Sylvester Esq. M.A. F.R.S.}

To cite this article: J.J. Sylvester Esq. M.A. F.R.S. (1844) LXXIV. On the Existence of absolute criteria for determining the roots of numerical equations, Philosophical Magazine Series 3, 25:168, 442-445, DOI: $10.1080 / 14786444408646082$

To link to this article: http://dx.doi.org/10.1080/14786444408646082

曲 Published online: 30 Apr 2009.

Submit your article to this journal $\sqsubset \pi$

Џ Article views: 2

Q View related articles $\square$ 
of the decomposition of nitric acid and persalts of iron, and finally the direct experiments of the reduction of gold, silver, platinum, palladium, nickel, copper, tin, and the decomposition of the persalts of iron and nitric acid inclosed within a tube, appear to set the question to rest, and to point out in the clearest manner that hydrogen is the cause of the reduction of the metals. All the experiments which I have detailed only add confirmation to the valuable researches of Faraday on Electro-Chemical Decomposition, published in the Philosophical Transactions. To the inquiring mind a question naturally arises as to whether the hydrogen reduces the metal directly from the metallic solution, or whether it reduces its oxide. 'The former opinion, from the above experiments, appears to be most worthy of credit, though should other facts be discovered to elucidate that astion, they will form subjects hereafter of a separate communication to this Society. It follows from these interesting experiments, that when a solution of metallic salt is subjected to the voltaic influence, the water is decomposed, oxygen passing one way and hydrogen the other; and that this hydrogen at the moment of decomposition on the negative plate performs the same part to sulphate of copper and other metallic salts that a piece of iron or zinc would to the same solutions.

LXXIV. On the Existence of Absolute Crileria for determi. ning the Roots of Numerical Equations. By J. J. Sylvester, E'sq., M.A., F.R.S.*

T WISH to indicate in this brief notice a fact which I be1 lieve has escaped observation hitherto, that there exist, certainly in some cases, and probably in all, infallible criteria for determining whether a given equation has all its roots rational or not.

In the equation of the second degree it is enough, in order that this may be the case, that the expression for the square of the difference of the roots shall be a perfect square; in other words, if $x^{2}-p x+q=0$ have its roots rational, $p^{2}-4 q$ must be not only a positive number (the condition of the roots being real), but that number must also be a complete square. In this case it is firther evident that $(p)$ must be either prime to $q$, or if uot, the greatest common measure of $p^{2}$ and $q$ must be a perfect square; but this condition is contained in the former, which is a sufficient criterion in itself.

If we now consider the equation of the third degree,

$$
x^{3}-p x^{2}+q x-r=0 \text {, }
$$

* Communicated by the Author. 
one condition is, that the product of the squared differences shall be a perfect square; in other words, the equation cannot have all its roots rational unless be a positive square number.

$$
p^{2} q^{2}-4 q^{3}-18 p q r-4 p^{3} r-27 r^{2}
$$

This remark is made at the end of the second supplement of Legendre's Theory of Numbers, and is indeed self-evident; and in like manner one condition may be obtained for an equation of any degree which is to have all its roots rational; but this is far from being the sole condition required.

In the equation of the third degree, however, one other condition, conjoined with that above expressed, will serve to determine infallibly whether all the roots are rational or not.

To obtain this condition, let us suppose that by making $3 x=y+p$ we obtain the equation

$$
y^{3}-\mathbf{Q} x-\mathrm{R}=0 \text {. }
$$

Calling the three roots of this new equation $\alpha, \beta, \gamma$ (all of which it is evident must be rational if those of the first equation are so), we have

$$
\begin{aligned}
\alpha+\beta+\gamma & =0, \\
\mathrm{Q}=-(\alpha \beta+\alpha \gamma+\beta \gamma) & =\alpha^{2}+\alpha \beta+\beta^{2}, \\
\mathrm{R}= & \alpha \beta \gamma .
\end{aligned}
$$

From the last two equations it is easily seen that if $k$ be any prime factor common to $\mathbf{Q}$ and $\mathbf{R}, k^{2}$ will be contained in $\mathbf{Q}$, and $k^{3}$ in $R$; or, in other words, $k$ will be a common measure of $\alpha, \beta, \gamma$.

We have therefore a second condition, that $9 q-3 p^{2}$ shall be a negative quantity, which is either prime to $2 p^{3}-9 q p+27 r$, or else so related to it, that the greatest common measure of the cube of the first and the square of the second is a perfect sixth power.

I now proceed to show the converse, that if these two conditions be both satisfied (and it will appear in the course of the inquiry that the first does not involve the second), the roots cannot help being all rational.

It is evident that the two conditions in question are tantamount to supposing that the roots of the proposed equation are linearly connected with those of another $z^{3}-Q z-R=0$ (by virtue of the assumption $3 x=k z+p$ ), where $\mathrm{Q}$ may be considered as prime to $R$; and where $4 Q^{3}-27 R^{2}$ is a perfect square.

Let now $4 Q^{3}-27 R^{2}=D^{2}$, then $D^{2}+27 R^{2}=4 Q^{3}$, or $\mathrm{D}^{2}+3(3 \mathrm{R})^{2}=4 \mathbf{Q}^{3}$.

Here, as $Q$ is prime to $R, D$ can have no common measure but 3 , with $3 \mathrm{R}$. 
444 On Criteria for determining Numerical Equations.

$1{ }^{\circ}$. Let $\mathbf{Q}$ be prime to $3 \mathrm{R}$.

Then putting $f^{2}+3 g^{2}=Q^{3}$, the complete solution of the equation immediately preceding is contained in the two systems.

$$
\begin{array}{ll}
\text { 1st. } & \mathrm{D}=2 f \quad 3 \mathrm{R}=2 g . \\
\text { 2nd. } & \mathrm{D}=(f \pm 3 g) \quad 3 \mathrm{R}=f \mp g,
\end{array}
$$

and for both systems,

$$
f \pm g \sqrt{-3}=(h+3 k \sqrt{-3})^{3} \text {. }
$$

The second system must therefore be rejected, for $g$ evidently contains 3 , and therefore $f=3 \mathrm{R} \pm g$ will contain 3 , and therefore $\mathbf{D}$ and therefore $\mathbf{Q}$ will do the same, contrary to supposition.

Hence

$$
\begin{aligned}
& \sqrt[3]{\frac{\mathrm{R}}{2} \pm \sqrt{-\left(\frac{\mathrm{Q}^{3}}{27}-\frac{\mathrm{R}^{2}}{4}\right)}}, \\
& =\sqrt[3]{\frac{\mathrm{R}}{2} \pm \frac{\mathrm{D}}{2}: \sqrt{-\frac{1}{27}}}, \\
& =\sqrt[3]{\frac{g}{3} \pm f \sqrt{-\frac{1}{27}}}, \\
& =\mp \frac{1}{3 \sqrt{-3}} \sqrt[3]{f \pm g \sqrt{-3}} \\
& =-\mathrm{K} \pm \frac{h}{3 \sqrt{-3}}=\lambda \pm \mu \sqrt{-3}
\end{aligned}
$$

and the three roots of the equation being

$$
\left\{\begin{array}{l}
(\lambda+\mu \sqrt{-3})+(\lambda-\mu \sqrt{-3}) \\
\frac{1 \pm \sqrt{-3}}{2}(\lambda+\mu \sqrt{-3})+\frac{1 \mp \sqrt{-3}}{2}(\lambda-\mu \sqrt{-3})
\end{array}\right.
$$

will evidently be all rational, which of course includes the necessity of their being also integer.

Again $2^{\circ}$, if we suppose that $Q$ does contain $3, D^{2}$ will contain 27 , and consequently $D$ will contain 9 ; and we shall have

$$
\mathrm{R}^{2}+3 \cdot\left(\frac{\mathrm{D}}{9}\right)^{2}=4 \cdot\left(\frac{\mathrm{Q}}{3}\right)^{3} \text {. }
$$

Here $R$ being prime to $\frac{D}{9}$, it may be shown, as in the last case, that the complete solution is

consequently

$$
\frac{\mathrm{R}}{2} \pm \frac{\mathrm{D}}{18} \sqrt{-3}=(h \pm k \sqrt{-3})^{3},
$$

$$
\sqrt[3]{\frac{\mathrm{R}}{2} \pm \sqrt{\frac{\overline{\mathrm{R}}^{2}}{4}-\frac{\mathrm{Q}^{3}}{27}}}=h \pm k \sqrt{\overline{-3}}
$$


and the three roots of the equation are

$2 h, h-3 k, h+3 k$ respectively,

and are therefore all rational.

Here it may be observed that the condition of $R$ being an even number, which we know, $a$ priori, is the case when all the roots are rational, is involved in the two more general conditions already expressed. It will now be evident that the first condition by no means involves the second, as it is perfectly easy to satisfy the equation $f^{2}+3 g^{2}=Q^{3}$ without supposing anything relative to $k$, the common measure of $f, g, Q$, except that it be itself of the form $\lambda^{2}+3 \mu^{2}$, which will give

$$
\left(\frac{f}{k}\right)^{2}+3 \cdot\left(\frac{g}{k}\right)^{2}=\left(\lambda^{2}+3 \mu^{2}\right)\left(r^{2}+3 s^{2}\right)^{3},
$$

an equation which can be solved in rational terms for all values of $\lambda, \mu, r, s$; and consequently the product of the squares of the differences of the roots may be a square, and at the same time the roots themselves may be irrational *.

I believe it will be found on inquiry that the equation $x^{n}-q x+r=0$ will always have two rational roots if

$$
(n-1)^{n-1} \cdot q^{n}-n^{n} \cdot r^{n-1}
$$

be a complete square, provided that $q$ be prime to $r$.

Furthermore, viewing the striking analogy of the general nature of the conditions of rationality already obtained, to those which serve to determine the reality of the roots of equations, I am strongly of opinion that a theorem remains to be discovered, which will enable us to pronounce on the existence of integer, as Sturm's theorem on that of possible roots of a complete equation of any degree: the analogy of the two cases fails however in this respect, that while imaginary roots enter an equation in pairs, irrational roots are limited to entering in groups, each containing two or MORE.

4 Park Street, Grosvenor Square,

November 7, 1844 .

\section{On some Photographic Phanomena. By George Shaw, Esq.†}

$\mathrm{T}$ is well known that the impression produced by light on a plate of silver, rendered sensitive by M. Daguerre's process, is wholly destroyed by a momentary exposure of the

* Thus then it appears that the total rationality of the roots of the equation $x^{3}-q x:-r=0$ may be determined by a direct method without having recourse to the 0 method of divisors to determine the roots themselves; the two conditions being that $49^{3}-27 r^{2}$ shall be a perfect square, and the greatest common measure of $q^{3}$ and $r^{2}$ a perfect sirth power.

$\uparrow$ Communicated by the Author. 